\title{
Induction of myasthenia by immunization against muscle-specific kinase
}

\author{
Kazuhiro Shigemoto, 1,2 Sachiho Kubo,, ${ }^{2}$ Naoki Maruyama, ${ }^{2}$ Naohito Hato, ${ }^{3}$ Hiroyuki Yamada, ${ }^{3}$ \\ Chen Jie, ${ }^{4}$ Naoto Kobayashi, ${ }^{4}$ Katsumi Mominoki, 5 Yasuhito Abe, ${ }^{6}$ \\ Norifumi Ueda, ${ }^{6}$ and Seiji Matsuda ${ }^{4}$
}

\begin{abstract}
${ }^{1}$ Department of Preventive Medicine, Ehime University School of Medicine, Ehime, Japan. 2Department of Molecular Pathology, Tokyo Metropolitan Institute for Gerontology, Tokyo, Japan. ${ }^{3}$ Department of Otolaryngology and ${ }^{4}$ Department of Integrated Basic Medical Science, Ehime University School of Medicine, Ehime, Japan. ${ }^{5}$ Department of Biological Resources, The Integrated Center for Science, Ehime University, Ehime, Japan. ${ }^{6}$ Department of Molecular Pathology, Ehime University School of Medicine, Ehime, Japan.
\end{abstract}

\begin{abstract}
Muscle-specific kinase (MuSK) is critical for the synaptic clustering of nicotinic acetylcholine receptors (AChRs) and plays multiple roles in the organization and maintenance of neuromuscular junctions (NMJs). MuSK is activated by agrin, which is released from motoneurons, and induces AChR clustering at the postsynaptic membrane. Although autoantibodies against the ectodomain of MuSK have been found in a proportion of patients with generalized myasthenia gravis (MG), it is unclear whether MuSK autoantibodies are the causative agent of generalized MG. In the present study, rabbits immunized with MuSK ectodomain protein manifested MG-like muscle weakness with a reduction of AChR clustering at the NMJs. The autoantibodies activated MuSK and blocked AChR clustering induced by agrin or by mediators that do not activate MuSK. Thus MuSK autoantibodies rigorously inhibit AChR clustering mediated by multiple pathways, an outcome that broadens our general comprehension of the pathogenesis of MG.
\end{abstract}

\section{Introduction}

Myasthenia gravis (MG) is an antibody-mediated autoimmune disease in which the nicotinic acetylcholine receptor (AChR) at neuromuscular junctions (NMJs) is the major autoantigen (1). AChR-specific antibodies are detected in $90 \%$ of nonimmunosuppressed patients with generalized MG. However, Hoch et al. found antibodies to a novel antigen, muscle-specific kinase (MuSK), in approximately $66 \%$ of patients with generalized MG that were lacking detectable AChR autoantibodies (seronegative MG) (2). Subsequent studies have reported MuSK antibody frequencies of 4-47.4\% in MG patients seronegative for AChR antibodies (3-9). MG patients with MuSK antibodies tend to develop severe facial weakness and bulbar symptoms, including dysphagia, dysarthria, and respiratory cirsis with some atrophy of facial muscles, that are often difficult to treat effectively with immunosuppressive therapies $(3,7)$. The pathogenic mechanisms of MG caused by AChR antibodies are well delineated, but pathogenicity has not been demonstrated for MuSK antibodies (10). Furthermore, no reports have described the induction of MG by immunization of animals with purified MuSK protein. The present study was undertaken to explore this issue. Here we describe the development of myasthenia and reduction of AChR density in rabbits immunized with the ectodomain of MuSK. The molecular pathogenesis of MG was further investigated using an in vitro assay of AChR clustering on myotubes that was mediated by MuSK antibodies.

MuSK is an AChR-associated transmembrane protein. During development of skeletal muscle, MuSK is initially required for

Nonstandard abbreviations used: BTX, $\alpha$-bungarotoxin; GalNAc, $N$-acetylgalactosamine; MG, myasthenia gravis; MuSK, muscle-specific kinase; MuSK-AP, chimeric protein of the MuSK ectodomain and human placental alkaline phosphatase; MuSK-Fc, chimeric protein of the MuSK ectodomain and the Fc region of human IgG1; NMJ, neuromuscular junction; VVA-B4, Vicia villosa agglutinin.

Conflict of interest: The authors have declared that no conflict of interest exists. Citation for this article: J. Clin. Invest. 116:1016-1024 (2006). doi:10.1172/JCI21545 organizing a primary synaptic scaffold to establish the postsynaptic membrane $(11,12)$. Prior to muscle innervation, AChR clusters form at the central regions of muscle fibers, creating an endplate zone that is somewhat broader than that in innervated muscle (13, 14). MuSK and rapsyn, which is a $43-\mathrm{kDa}$, membrane-associated cytoplasmic protein, must be expressed before the endplate zone forms (11,15-17). Subsequent contact of the motor-neuron growth cone with the muscle extinguishes extrasynaptic AChR clusters, resulting in a narrow, distinct endplate zone in the midmuscle that is marked by a high density of AChR clustering $(13,14)$. In this step, agrin released from motoneurons activates MuSK and redistributes AChR clusters to synaptic sites $(13,14,17-20)$. Therefore the formation of NMJs either in the absence or presence of agrin requires the expression of MuSK at the endplate membrane.

The extracellular segment of MuSK comprises 5 distinct domains, i.e., 4 immunoglobulin-like domains and 1 cysteinerich region (21-25). All 5 domains are conserved in Torpedo spp, mice, humans, rats, Xenopus, and chickens. MuSK exerts its multiple effects through interaction of the extracellular domains with other molecules. Transfection of $\mathrm{MuSK}^{-/-}$myotubes with a series of mutant MuSK constructs demonstrated that the aminoterminal immunoglobulin-like domain is required for activation by agrin (26). The extracellular domains of MuSK interact with rapsyn-AChR complexes $(27,28)$. Although the synaptic membrane in adult muscle appears to be macroscopically stable, in vivo studies have shown that synaptic AChRs intermingle completely over a period of approximately 4 days, and that many extrasynaptic AChRs are incorporated into the synapse at the mature NMJs $(29,30)$. In addition, RNA interference targeting MuSK in adult mammalian muscle in vivo induced the disassembly of postsynaptic AChR clusters, with effects ranging from fragmentation to disappearance (31). Hence the mechanisms of NMJ formation by MuSK are also likely to be important for the maintenance of AChR clusters at the NMJs. 

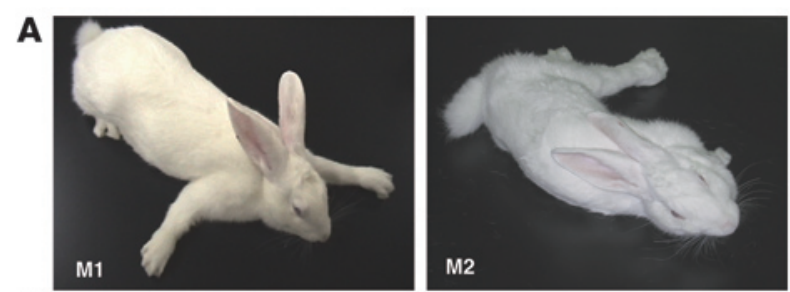

B
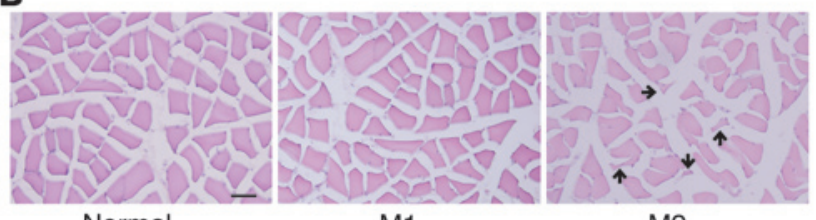

Normal

M1

M2

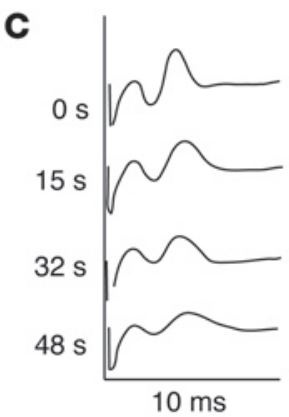

In cultured myotubes, AChR clustering is induced by laminin-1 and the $N$-acetylgalactosamine-specific (GalNAc-specific) lectin Vicia villosa agglutinin (VVA-B4) without activation of MuSK (32-36). Neither the receptor nor the activation mechanisms of AChR clustering induced by agrin-independent inducers has been identified with certainty. Even so, these mechanisms may also play important roles in the maintenance of NMJs via agrin-independent pathways and in their formation, as shown by genetic studies $(13,14)$. The data we present herein demonstrate that MuSK autoantibodies inhibit AChR clustering by agrin itself and also by all known agrin-independent pathways.

\section{Results}

Immunization with purified MuSK protein causes flaccid weakness in rabbits. Rabbit antibodies were raised against a purified chimeric protein composed of the MuSK ectodomain and the Fc region of human IgG1 (MuSK-Fc). All of 4 recipient rabbits manifested flaccid weakness after 3 or 4 repeated injections with MuSK-Fc. Three of these rabbits developed flaccid weakness within 3 weeks after the last injection of MuSK protein, and the fourth rabbit manifested flaccid weakness 9 weeks after the third injection. Two rabbits that manifested flaccid weakness (M1 and M2 paretic rabbits) are shown in Figure 1A and Supplemental Movies 1 and 2 (supplemental material available online with this article; doi:10.1172/ JCI21545DS1). Two of 4 paretic rabbits developed severe exhaustion (Figure 1A and Supplemental Movie 2; M2 paretic rabbit). Histological studies of the muscle tissues in the paretic rabbits revealed that the angular atrophic muscle fibers in the M2 paretic rabbit were intermingled with normal fibers, whereas the M1 rabbit had only subtle changes in the muscles (Figure 1B). No muscle regeneration was observed in $\mathrm{M} 1$ and $\mathrm{M} 2$ paretic rabbits (Figure 1B). The histological changes of the atrophic muscle fibers

\section{Figure 1}

Rabbits manifest MG-like paresis after immunization with MuSK protein. (A) Two rabbits, representative of the 4 animals that showed myasthenia, manifested myasthenic weakness after immunization with the recombinant MuSK protein. After 3 injections of MuSK protein, M1 and M2 rabbits manifested flaccid weakness within 3 and 9 weeks, respectively. The M2 rabbit developed severe exhaustion with muscle weakness. (B) Cross sections from the soleus muscles of 2 paretic (M1 and M2) and 1 normal rabbit were stained with $\mathrm{H} \& \mathrm{E}$. Muscle fibers in the M1 paretic rabbit showed only subtle changes in shape and smallness, whereas an atrophy of muscle fibers in the M2 paretic rabbit was observed as small angular fibers (arrows). Scale bar: $50 \mu \mathrm{m}$. (C) Electromyograms recorded from the M1 paretic rabbit. The retroauricular branch of the facial nerve was continuously stimulated by a constant-current stimulator, which delivered square-wave pulses of $0.1 \mathrm{~ms}$ at $20 \mathrm{~Hz}$, and the compound muscle action potential (the second peak observed on the oscilloscope screen was recorded at the indicated time points during stimulation) showed a decremental pattern, consistent with MG.

observed in the M2 paretic rabbit can result from MG, reduced mechanical activity of muscles, or cachexia (37).

These results suggest that the muscle weakness was caused by a disturbance of neuromuscular transmission due to the inhibition of MuSK functions in mature NMJs. This possibility was investigated first by an electromyographic study (38). Repetitive nerve stimulation (RNS) at a rate of $20 / \mathrm{s}$ in a paretic animal elicited a decremental response in compound muscle action potential (CMAP), characteristic of MG (Figure 1C). However, the CMAP in a normal rabbit did not change significantly during RNS at the same rate (data not shown). This result is consistent with the M1 rabbit having a disorder of the postsynaptic membrane as the cause of its weakness following immunization with a fragment of MuSK protein.

Reduction of AChR clustering at NMJs in paretic rabbits. Our second objective was to examine the NMJs of soleus muscles in 2 paretic (M1 and M2) and 3 normal rabbits (N1, N2, and N3) by fluorescence microscopy after applying a rhodamine-conjugated AChR antagonist, $\alpha$-bungarotoxin (BTX). For semiquantitative measurement of the size and density of AChR clustering at NMJs, we determined an appropriate concentration of rhodamine-conjugated BTX by using serial dilutions of this reagent for application to frozen sections of soleus muscles (Figure 2A). A digital camera recorded images of the AChR clustering. Ten AChR clusters of NMJs were randomly chosen from the left and 10 from the right soleus muscles, after which the sizes and ODs were measured using NIH Image analysis software with the unprocessed digitized images (Supplemental Figure 1). In agreement with the electromyographic studies described above, the area and intensity of AChR fluorescence at the NMJs in both M1 and M2 paretic rabbits were significantly reduced compared with normal rabbits (area: M1, $67.9 \pm 58.2 \mu \mathrm{m}^{2} ; \mathrm{M} 2,75.0 \pm 55.9 \mu \mathrm{m}^{2}$; compared with $\mathrm{N} 1,303.9 \pm 125.9 \mu \mathrm{m}^{2} ; \mathrm{N} 2,387.7 \pm 163.0 \mu \mathrm{m}^{2} ; \mathrm{N} 3,395.4 \pm 150.0$ $\mu \mathrm{m}^{2}$; density: M1, $6219 \pm 4659$ OD; M2, $6889 \pm 4259$ OD; compared with N1, 17,770 \pm 7,693 OD; N2, 23,259 \pm 9,046 OD; N3, 23,870 \pm 9,997 OD; Figure 2B).

Specific binding of antibodies to MuSK. As we found that muscle weakness caused by a disorder in the postsynaptic membrane was induced in rabbits via immunization with a purified MuSK protein, we next examined the ability of immune serum from paretic rabbits to react specifically with MuSK. To test whether MuSK 
A
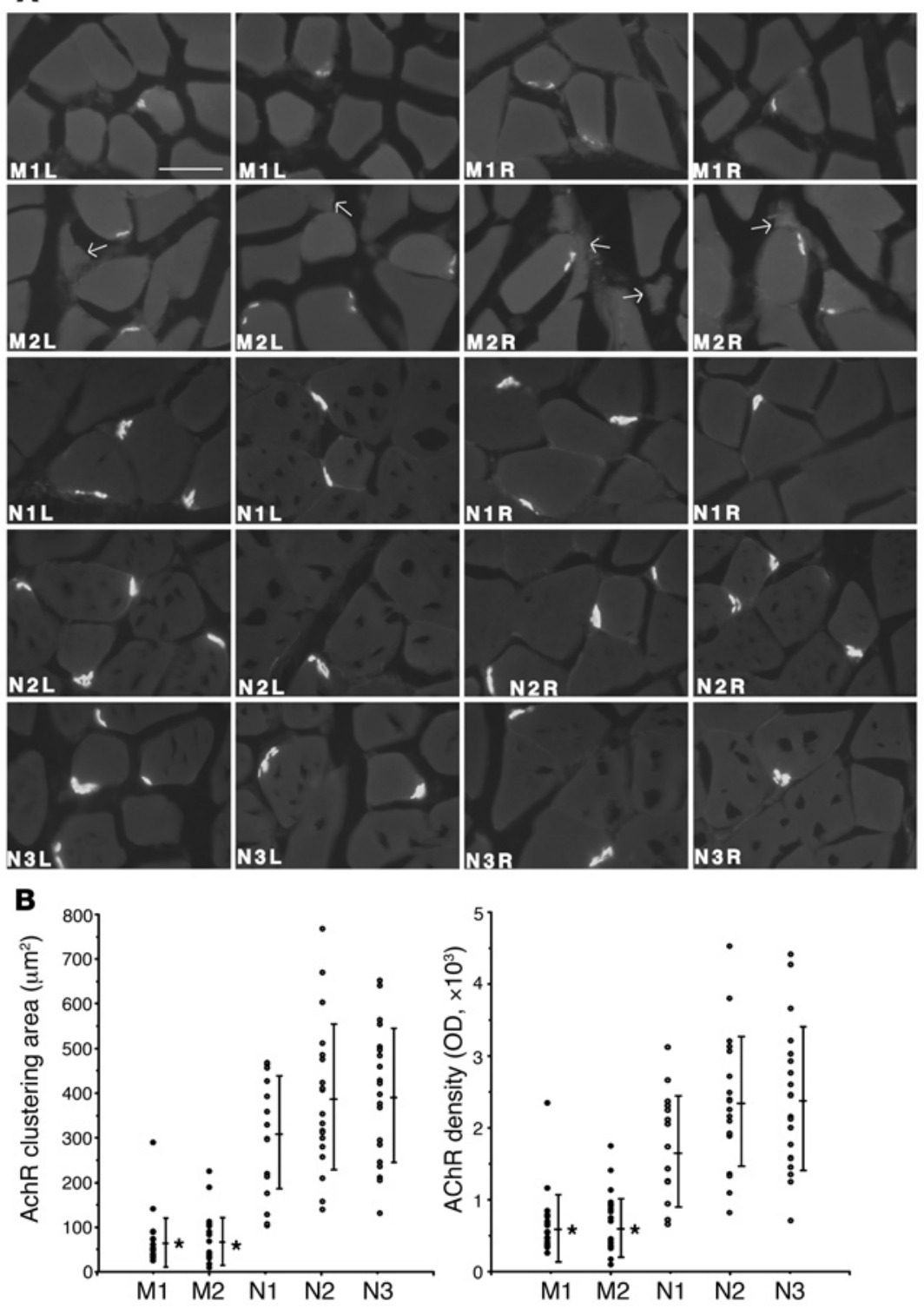

\section{Figure 2}

Reduction of the size and density of AChR clusters at the NMJs in paretic rabbits. (A) Cross sections from the soleus muscles of 2 paretic (M1 and M2) and 3 normal rabbits (N1, N2, and N3) were stained with $10 \mathrm{nM}$ rhodamine-conjugated BTX. Bright crescents of bound BTX, indicative of endplate AChR, were smaller and less intense in the paretic rabbits' muscle fibers than in those of the normal rabbits. Arrows indicate the small angular fibers in M2 soleus muscles. L, left; R, right. Scale bar: $50 \mu \mathrm{m}$. (B) Images of $10 \mathrm{AChR}$ clusters at NMJs in the right and 10 in the left soleus muscles of the paretic and normal rabbits were randomly recorded by a digital imaging camera. Quantification of the area and intensity of AChR clustering in the unprocessed images were measured using NIH Image software. Bars indicate mean \pm SD. ${ }^{*} P<0.01$ versus normal rabbits.

the clustering of AChR on the surfaces of $\mathrm{C} 2 \mathrm{C} 12$ myotubes in the absence of agrin. Therefore, the effect of MuSK antibodies on the tyrosine phosphorylation of MuSK was assessed here in C2C12 cell cultures. After a 30-minute incubation with MuSK antibodies, MuSK protein was immunoprecipitated from detergent extracts of the cells by using an antiserum against its intracellular domain. Tyrosine-phosphorylated MuSK was detected in Western blots by using phosphotyrosine-specific antibodies (Figure 4A). This antibody-induced autophosphorylation was completely inhibited by absorption with an excess of MuSK-AP before the addition of MuSK antibodies to the C2C12 myotube cultures. Thus the MuSK antibodies specifically activated autophosphorylation by inducing dimerization of the MuSK molecules in the absence of agrin, as shown previously using $\mathrm{N}$-terminal-specific MuSK antibodies.

The downstream cascade of MuSK also leads to tyrosine phosphorylation of the AChR $\beta$ subunit $(19,40,41)$. Therefore we next sought to deter-

antibodies could be used to detect MuSK in Western blots, a chimeric protein that combined the MuSK ectodomain with human placental alkaline phosphatase (MuSK-AP) was transfected into COS-7 cells. As expected, proteins representing the chimeric products $(\sim 130 \mathrm{kDa})$ were secreted into the culture medium (Figure $3 \mathrm{~A})$. The immune serum did not react with any of the AChR subunits precipitated from a lysate of $\mathrm{C} 2 \mathrm{C} 12$ myotubes, as determined using biotinylated BTX and streptavidin-agarose. In contrast, the AChR $\beta$ subunit was detected in the same precipitate when using a monoclonal antibody (data not shown). In addition, immunohistochemical analysis showed that MuSK antibodies reacted with native MuSK molecules and colocalized with agrininduced AChR clusters on the surfaces of differentiated C2C12 myotubes (Figure 3B). Therefore a subset of MuSK antibodies reacts specifically with MuSK molecules on the cell surface.

Activation of MuSK by autoantibodies against MuSK. Hopf and Hoch (39) reported that rabbit polyclonal antibodies against the MuSK N-terminal sequence induced the activation of MuSK and mine whether AChRs are tyrosine phosphorylated in response to MuSK activation by the MuSK antibodies. C2C12 myotubes were exposed to biotinylated BTX to identify AChRs in phosphotyrosine immunoblots. After exposure of the myotubes to MuSK antibodies for 30 minutes, tyrosine phosphorylation of the AChR $\beta$ subunit was detected at a level similar to that induced by agrin (Figure 4B). AChR phosphorylation was inhibited when the antibodies were absorbed with an excess of MuSK-AP prior to addition to cultured $\mathrm{C} 2 \mathrm{C} 12$ myotubes, which demonstrates that the MuSK antibodies specifically induce AChR $\beta$ subunit phosphorylation in the downstream cascade of MuSK. Antibodies against the intracellular domain of the MuSK protein did not phosphorylate either MuSK or the AChR $\beta$ subunit (data not shown).

Inbibition of spontaneous ACbR clustering by antibodies against the MuSK ectodomain. MuSK antibodies induced tyrosine phosphorylation of both MuSK and the AChR $\beta$ subunit, whereas AChR clustering at the NMJs of paretic rabbits was reduced. Therefore, we next tested the ability of MuSK antibodies to stimulate AChR 


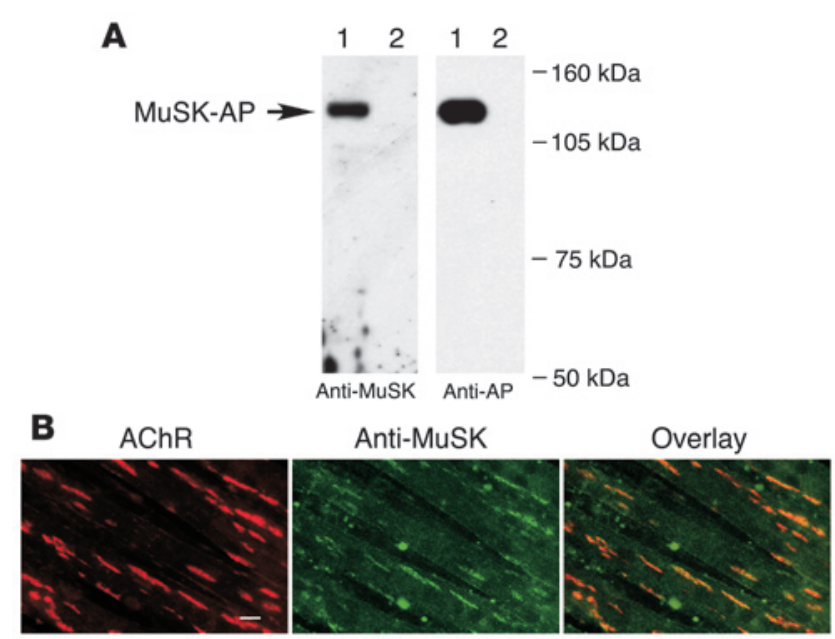

clustering in the absence of agrin following MuSK autophosphorylation. Overnight incubation of C2C12 myotubes with MuSK antibodies in the absence of soluble agrin and labeling of AChR with rhodamine-conjugated BTX revealed that the MuSK antibodies inhibited spontaneous AChR clustering; that is, only a small number of AChR clusters formed in the myotubes in the absence of additional stimulation (Figure 5, A and B). However, AChR clustering was not blocked by antibodies against the MuSK intracellular domain (data not shown). Taken together, these results suggest that MuSK antibodies do not induce AChR clustering. Instead, they interfere with spontaneous AChR clustering by interacting with MuSK molecules in C2C12 myotubes, despite the activation of MuSK autophosphorylation.

Inbibition of agrin-induced AChR clustering by MuSK antibodies. Agrin induces clustering of AChR in C2C12 myotubes following MuSK autophosphorylation $(17,19,20)$. This cascade of events is a major feature of AChR clustering at the NMJs after innervation by motoneurons $(17,36,42,43)$. In a previous study, Hoch et al. observed that the MuSK antibodies of MG patients inhibited agrin-induced AChR clustering (2), whereas we found that MuSK antibodies from paretic rabbits activated MuSK. It was therefore of interest to determine whether MuSK antibodies could interfere with agrin-induced AChR clustering in $\mathrm{C} 2 \mathrm{C} 12$ cells as they did in spontaneous clustering. Indeed, agrin-induced clustering of AChR was strongly blocked in the presence of MuSK antibodies, whereas absorption of the antibodies with soluble MuSK-AP fusion products prevented this blocking effect (Figure 6, A and B). This result clearly demonstrates that the MuSK antibodies are responsible for inhibiting the formation of agrin-induced AChR clustering, in contrast to the N-terminal-specific MuSK antibodies, which induce AChR cluster formation in C2C2 myotubes (39).

Inbibition of agrin-independent AChR clustering by antibody against the MuSK ectodomain. Since MuSK is necessary for the signaling and effector mechanisms of agrin-independent AChR clustering at the NMJs in vivo $(13,14)$, we examined the ability of MuSK antibodies to inhibit AChR clustering induced by agrin-independent stimuli such as laminin-1 and VVA-B4. Incubation of laminin-1 with MuSK antibodies strongly blocked AChR clustering as well as spontaneous and agrin-induced AChR clustering (Figure 6, A and B) on C2C12 cells. After absorption of the anti-

\section{Figure 3}

MuSK autoantibodies specifically bind MuSK. (A) Detection of secreted MuSK-AP from COS-7 cells by Western blot analysis with MuSK autoantibodies. The culture supernatants from COS-7 cells transfected with MuSK-AP (lane 1) or vector alone (lane 2) were immunoblotted with the MuSK antibodies, and the same immmunoblots were reprobed with an anti-alkaline phosphatase (anti-AP) antibody. (B) Specific binding of MuSK autoantibodies to MuSK in C2C12 myotubes. Myotubes were treated with agrin ( $1 \mathrm{nM}$ for $18 \mathrm{~h}$ ) and double labeled, first with rhodamine-conjugated BTX (left), and then with MuSK antibodies followed by fluorescein-conjugated secondary antibodies (middle). AChRs and MuSK colocalized, as shown by the overlay of red and green fluorescence (right). Green blotches are artifact. Scale bar: $20 \mu \mathrm{m}$.

bodies with soluble MuSK-AP fusion products, AChR clustering in response to laminin-1 was restored (Figure 6, A and B). Thus the blocking effect was caused by these antibodies specific for the MuSK ectodomain, as was observed for agrin-induced clustering. In addition, treatment of C2C12 cells with VVA-B4 and MuSK antibodies totally blocked AChR clustering, whereas absorption of the antibodies with soluble MuSK-AP protein prevented this blocking effect (Figure 6, A and B). Therefore blocking by the antibodies was also specific for the MuSK ectodomain. These results indicate that the interaction of MuSK antibodies with MuSK molecules in myotubes leads to a reduction of AChR clustering via an agrin-independent pathway.

Blocking of neuraminidase-stimulated AChR clustering by MuSK antibodies. Treatment of $\mathrm{C} 2 \mathrm{C} 12$ cells with neuraminidase increases both the intensity of VVA-B4 staining and the number of AChR clusters compared with spontaneous clustering $(34,43)$. Since AChR clustering caused by neuraminidase treatment may involve the same signaling mechanism as that induced by VVA-B4, the ability of MuSK antibodies to inhibit this response was examined. We con-

A

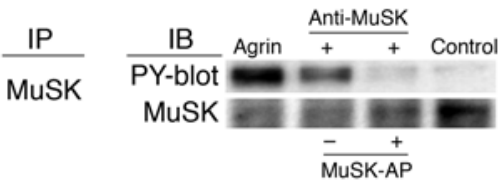
B IP Biotinylated BTX

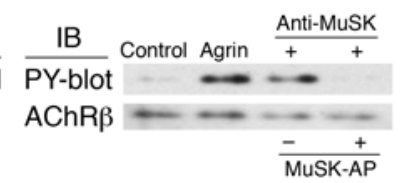

\section{Figure 4}

MuSK antibodies specifically activate MuSK and the downstream cascade. Specific phosphorylation of MuSK and AChR $\beta$ subunit (AChR $\beta)$ by MuSK antibodies. C2C12 myotubes were treated with agrin or MuSK antibodies for 30 minutes and then immunoprecipitated with (A) MuSK antibodies or (B) biotinylated BTX using streptavidin-Sepharose. Immunoblots of immunoprecipitates were probed with antibodies to $\operatorname{MuSK}(\mathbf{A}), A C h R \beta(B)$, or phosphotyrosine (PY, A and B). Both MuSK and $A C h R \beta$ were phosphorylated after the treatment of agrin or MuSK antibodies. Phosphorylation of MuSK and AChR $\beta$ was inhibited by the absorption of MuSK antibodies with MuSK-AP protein. 

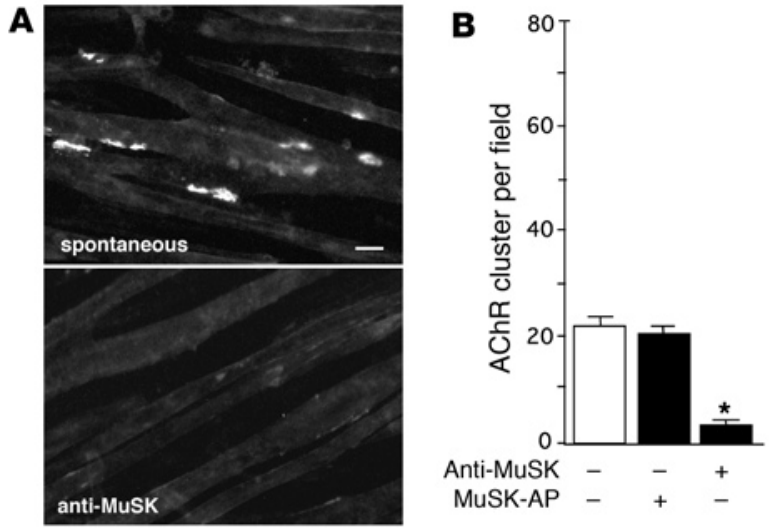

firmed that neuraminidase treatment stimulated AChR clustering in C2C12 myotubes. That is, overnight incubation of C2C12 cells with $0.1 \mathrm{U} / \mathrm{ml}$ neuraminidase resulted in a 3 -fold increase in the number of AChR clusters per field (Figure 7, A and B). When the C2C12 cells were incubated with MuSK antibodies and neuraminidase for 16 hours, AChR clustering was blocked efficiently, as was the clustering induced by VVA-B4. Again, absorption with MuSKAP prevented blocking (Figure 7, A and B), which demonstrates that the inhibition of AChR clustering is caused by a direct effect of the MuSK antibodies. In summary, MuSK antibodies from paretic rabbits inhibit the AChR clustering in myotubes induced by all known agrin-independent stimuli as well as by agrin itself.

\section{Discussion}

MuSK autoantibodies are found in $4-70 \%$ of patients with generalized MG who lack detectable AChR autoantibodies (seronegative MG) (2-9); therefore, we investigated the pathogenicity of MuSK autoantibodies, as reported here. Our results demonstrate that immunization of rabbits with MuSK ectodomain protein caused myasthenic weakness and produced electromyographic findings that were compatible with a diagnosis of MG and consistent with a significant reduction of AChR clustering at NMJs. Additionally, MuSK antibodies specifically inhibited in vitro AChR

\section{Figure 6}

Inhibition of agrin-induced and agrin-independent AChR clustering by MuSK antibodies. (A) C2C12 cells were treated with agrin, laminin-1, or VVA-B4. AChR clusters were stained with rhodamine-conjugated BTX. AChR clustering induced by agrin, laminin-1, and VVA-B4 was inhibited in the presence of MuSK antibodies. This inhibition was blocked by absorption of the MuSK antibodies with MuSK-AP before treatment of the cells. Scale bar: $20 \mu \mathrm{m}$. (B) Quantification of the inhibitory activity of the MuSK antibodies confirmed that they significantly inhibited agrin-, laminin-1-, and VVA-B4-induced AChR clustering. Preabsorption of the MuSK antibodies with MuSK-AP significantly blocked inhibition. Values represent mean \pm SEM of 10-15 fields for each of the 2 experiments per treatment. ${ }^{*} P<0.01$ versus similar treatment without MuSK antibodies; ${ }^{\#} P<0.01$ versus similar treatment without preabsorption; ANOVA.

\section{Figure 5}

Inhibition of spontaneous AChR clustering by MuSK antibodies. (A) AChR clusters on $\mathrm{C} 2 \mathrm{C} 12$ myotubes were stained with rhodamine-conjugated BTX with or without MuSK antibodies. Scale bar: $20 \mu \mathrm{m}$. (B) Quantification of the inhibitory activity of the MuSK antibodies showed that these antibodies provided significant inhibition of spontaneous AChR clustering ( ${ }^{*} P<0.01$, ANOVA). Values represent mean \pm SEM of 10-15 fields for each of the 2 experiments per treatment. clustering responses to all known stimuli, including those that act via agrin-independent pathways. Surprisingly, when applied to C2C12 muscle cells, MuSK antibodies initiated the phosphorylation of MuSK receptors and the subsequent phosphorylation of $\mathrm{AChR}$ in the absence of agrin, despite strongly inhibiting spontaneous AChR clustering. Furthermore, the MuSK antibodies inhibited AChR clustering induced by agrin. The possibility that MuSK antibodies contain a component that reacts with a receptor that stimulates inhibitory signals for AChR clustering seems unlikely,
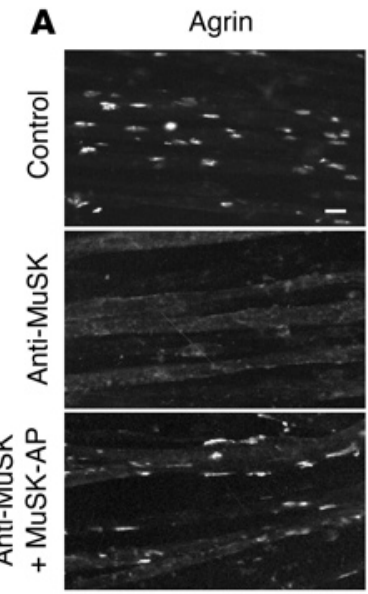

$\mathbf{B}$

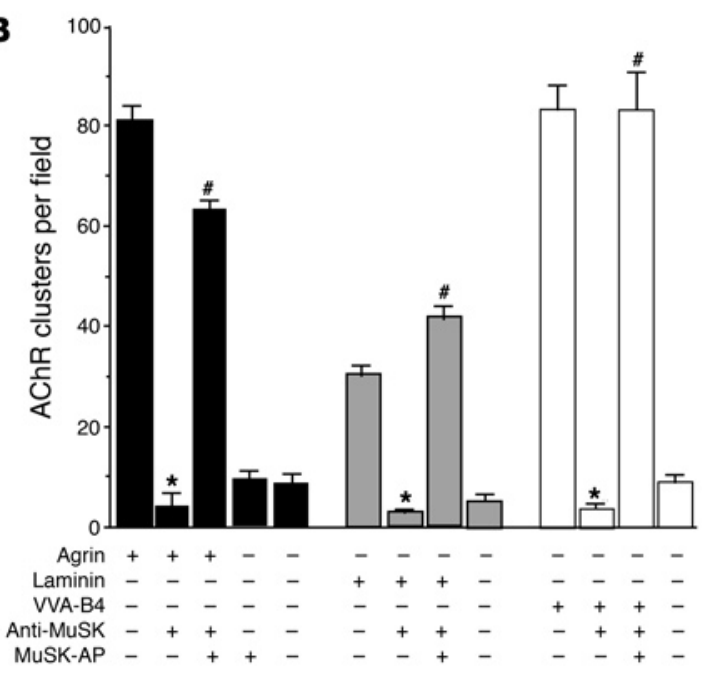




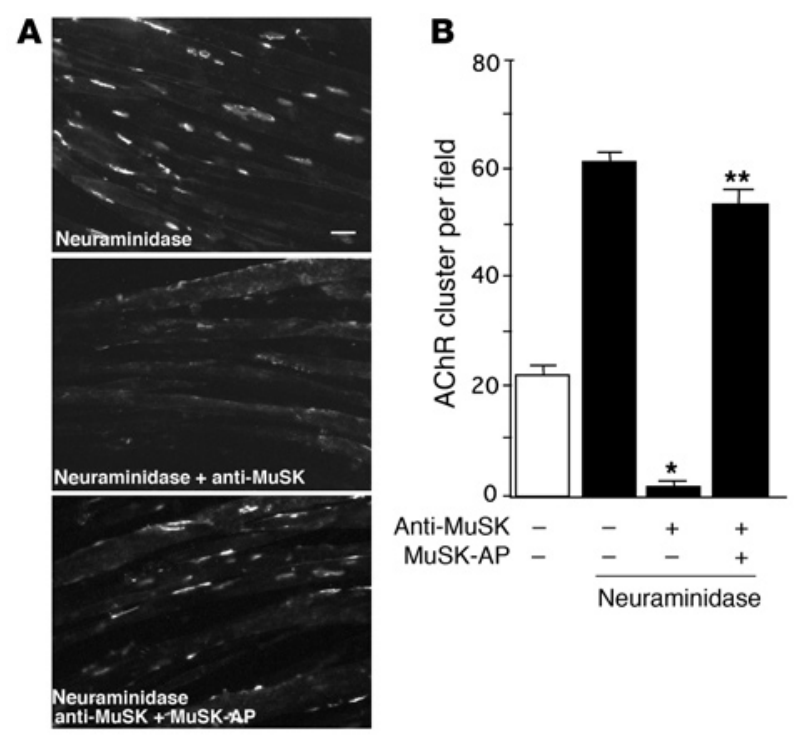

since the ability of these antibodies to inhibit AChR clustering was abolished by absorption with a purified chimeric recombinant protein of MuSK. Hoch et al. have shown that plasma containing MuSK antibodies derived from patients with generalized MG who lack detectable AChR autoantibodies causes modest AChR clustering, whereas the same plasma inhibits agrin-induced AChR clustering (2). Thus, it is conceivable that a subset of MuSK antibodies expresses a strong inhibitory activity that blocks spontaneous AChR clustering, whereas other subsets of MuSK antibodies, such as those directed against the $\mathrm{N}$ terminus of the ectodomain, have a modest capacity to induce AChR clustering (39). An alternative possibility is that the longer incubation time with the MuSK antibodies used in the study of Hoch et al. caused the reverse effect by downregulating scaffold molecules required for AChR clustering. The effects on spontaneous clustering of MuSK antibodies reported by Hoch et al. were observed after 5 hours of incubation, whereas our analysis was carried out after a 16-hour incubation with the antibodies (2).

Neither the receptor responsible for nor the activation mechanism of AChR clustering induced on myotubes by laminin-1 is known. However, the signaling mechanisms of agrin and laminin-1 converge at a site downstream from $\operatorname{MuSK}(19,35)$. Another agrinindependent stimulus, VVA-B4, recognizes terminal $\beta$-linked GalNAc residues and binds at the NMJ (33). Moreover, even in the absence of rapsyn-AChR complexes, agrin induces MuSKbased scaffolding, including the provision of binding sites for VVA-B4 on myotubes (12). Thus VVA-B4 receptors acting in concert with MuSK appear to play a key role in AChR clustering $(12,44)$. However, MuSK is not a receptor for VVA-B4, as demonstrated by the absence of O-linked carbohydrates providing O-linked GalNAc residues in MuSK molecules (45). Neuraminidase treatment also induces AChR clustering on myotubes by its action on terminal $\beta$-linked GalNAc residues (34). By eliminating sialic acid from glycoconjugates in the membranes, neuraminidase increases the exposure of terminal $\beta$-linked GalNAc residues that bind to the GalNAc-specific lectin VVA. We believe our observation that MuSK-specific antibodies strongly inhibited AChR clustering induced by all agrin-independent pathways as well as by agrin to be unprecedented.

\section{Figure 7}

Inhibition of neuraminidase-induced AChR clustering by MuSK antibodies. (A) $\mathrm{C} 2 \mathrm{C} 12$ cells were treated with Clostridium perfringens neuraminidase. AChR clusters were stained with rhodamine-conjugated BTX. AChR clustering induced by neuraminidase was inhibited in the presence of the MuSK antibodies. This inhibition was blocked by absorption of the MuSK antibodies with MuSK-AP before treatment of the cells. Scale bar: $20 \mu \mathrm{m}$. (B) Quantification of the significant inhibition of neuraminidase-induced AChR clustering by MuSK antibodies. Inhibition was significantly blocked by preabsorption of the MuSK antibodies with $6.5 \mathrm{nM}$ MuSK-AP. Values represent mean \pm SEM of 10-15 fields for each of the 2 experiments per treatment. ${ }^{*} P<0.01$ versus treatment without MuSK antibodies; ${ }^{* *} P<0.01$ versus treatment without preabsorption; ANOVA.

Current knowledge offers several possible mechanisms for the global inhibition of this signaling pathway. Prior to innervation, MuSK is essential for organizing a primary synaptic scaffold and initiating postsynaptic membrane maturation events $(11,12,16)$. These mechanisms may also be necessary for the maintenance of AChR clusters at mature NMJs, where AChR clusters undergo rapid remodeling $(29,30)$. Therefore, antibody to MuSK could directly prevent scaffold formation, which may be requisite for AChR clustering taking place in mature NMJs, as shown by RNA interference experiments (31). For example, the interaction of MuSK and rapsyn-AChR complexes is required for AChR clustering by agrin $(11,46)$. A second possible mechanism governing the inhibition of AChR clustering is an antibody-induced decrease of MuSK expression at the cell surface, which might also prevent scaffolding. In addition, the antibody could act to internalize other associated components required for agrin-dependent or agrin-independent AChR clustering. Through either mechanism, a subset of MuSK antibodies could interfere with AChR clustering even when stimulated by a different subset of MuSK antibodies and subsequently cause the extrajunctional spread of AChRs at mature NMJs.

Finally, the ability to block AChR clustering is not the sole function of MuSK antibodies, since sera containing MuSK antibodies obtained from patients with MG also inhibited agrindependent and agrin-independent AChR clustering on myotubes (our unpublished results). The experimental model developed here (Figure 8 ) is based on the use of MuSK antibodies derived from an animal whose autoimmune response impaired neuromuscular transmission. This model will facilitate further progress in resolving the pathogenic basis of MG at the molecular level and identifying other aspects of pathogenicity available to these autoantibodies in vivo.

\section{Methods}

Isolation of MuSK cDNA clones. Mouse MuSK cDNA clones were isolated by constructing a mouse $\mathrm{C} 2 \mathrm{C} 12$ myotube cDNA library in the $\lambda$ ZAPII vector (Stratagene) (47). The library was screened with a human MuSK cDNA probe amplified by reverse transcription PCR, together with a set of primers and total RNA from human muscle (Stratagene). The following primers were designed based on the sequence of the human MuSK cDNA: 
A

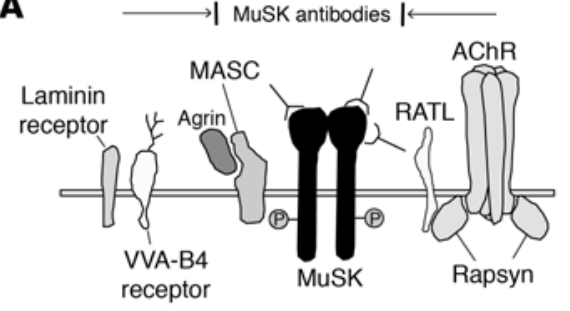

B

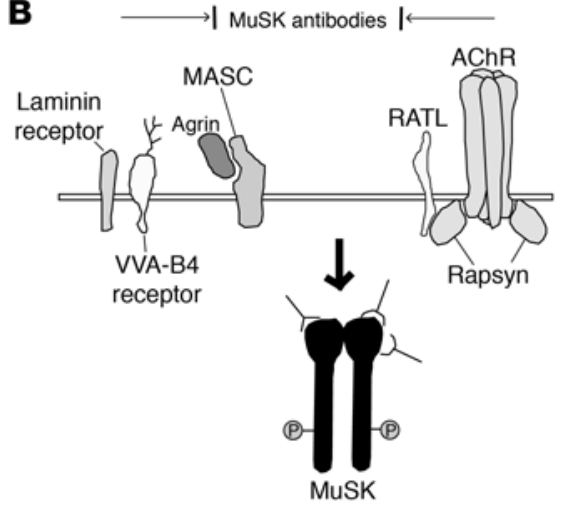

Figure 8

Models of MuSK antibody inhibition of AChR clustering on myotubes. (A) MuSK antibodies cover the ectodomain of MuSK and inhibit interactions with other scaffolding molecules required for $\mathrm{AChR}$ clustering, including myotube-associated component (MASC), rapsyn-associated transmembrane linker (RATL), and receptors for VVA-B4 and laminin. MASC is a hypothetical molecule that is required for agrin binding and the activation of MuSK. RATL, also a hypothetical molecule, mediates the association of rapsyn with the extracellular domain of MuSK. (B) MuSK antibodies induce the internalization of MuSK in the presence or absence of associated molecules. The lack of MuSK on the surfaces of myotubes inhibits the effects of scaffolding and AChR clustering.

5'-GTTCTCCAGAAGGAACTTCGTCCTGC-3' and 5'-CCGTGCAGCGCAGTAAATGCCATCATC-3' (25). Sequences were identified using the ABI 310 DNA sequencer and BigDye Terminator v1.1 Cycle Sequencing Kit (Applied Biosystems). Sequence analysis was carried out using the GCG package of the Human Genome Center, Institute of Medical Science of the University of Tokyo.

Recombinant proteins. A MuSK-AP fusion protein was produced by amplifying the MuSK cDNA from nucleotide 2 in the $5^{\prime}$ untranslated region to nucleotide 1337 (GenBank/EMBL accession number AY360453) using $\mathrm{PCR}$, adding terminal BspEI sites, and inserting the construct into the $B s p$ EI site of the APtag-2 vector (48). The fusion protein expression construct, which consisted of the MuSK ectodomain and the Fc region of human IgG1, was generated with the same strategy used for the alkaline phosphatase construct. The MuSK cDNA from nucleotides 2 to 1337 was amplified by PCR; an artificial splicing donor signal (GTGAGT) was added at the $3^{\prime}$ terminus of the $\mathrm{CDNA}$, and the construct was inserted between the SalI and SpeI sites of the vector pEF-Fc (gift from Y. Yoshihara, RIKEN Brain Science Institute, Wako, Saitama, Japan). We generated expression vectors carrying the secreted, soluble form of mouse neural agrin (C-Ag4,19; $y=4$, $Z=19$ ), using PCR to amplify the 1.5 -kb C-terminal half of an alternative isoform of mouse agrin cDNA, which contains 19 amino acids at the $z$ site, and using the first-strand cDNA of the mouse spinal cord (49). The following primers were designed based on the sequence of mouse agrin: 5'-GGGGATCCTGGCCGCTTTGGCCCAACTTGTGCAGATG-3' and 5'-GCTCTAGAGAGAGTGGGGCAGGGTCTTAGCTC-3' (50).

The amplified agrin cDNA, with added terminal BamHI and $\mathrm{XbaI}$ sites, was inserted between the BamHI and XbaI sites of the pSecTag vector (Invitrogen Corp.), which contains the ER signal sequence of the mouse Igk gene (47) and a myc/his-tag sequence at the $3^{\prime}$ site. COS-7 cells were transfected by using Fugene- 6 reagent (Roche Molecular Biochemicals) according to the manufacturer's instructions. The secreted recombinant agrin (C-Ag4,19) was concentrated with centrifugal filter devices (Millipore), and the secreted recombinant MuSK-AP and MuSK-Fc proteins were purified by using alkaline phosphatase monoclonal antibodies coupled to agarose (SigmaAldrich) and protein A-Sepharose (Amersham Biosciences), respectively, by standard methods (48). Agrin protein was purified from the supernatant of transfected COS-7 cells in a Sephadex G-200 column (Amersham Biosciences). Peak fractions that contained agrin were identified by Western blot analysis with anti-myc antibodies (Invitrogen Corp.), then pooled and concentrated. The purity of the recombinant proteins was determined by SDS-PAGE with silver staining. The concentration of recombinant proteins was determined by using the BCA Protein Assay kit (Pierce Biotechnology) with BSA as the standard. The relative band intensities for MuSK-AP, MuSK-Fc, agrin $(z=19)$, and known amounts of BSA were compared on Coomassie-stained gels as an independent measure.

Animal experiments and antibodies. The Ehime University Animal Care and Use Committee approved all of the procedures used in the animal experiments. Antiserum against the MuSK ectodomain (the MuSK antibodies) was raised by repeatedly injecting New Zealand White rabbits with $100-400 \mu \mathrm{g}$ of protein A-Sepharose-purified MuSK-Fc. Three of the 4 injected rabbits manifested fatigable muscle weakness after 3 rounds of immunization. One rabbit developed the symptoms after the fourth round of immunization. Electromyograms were recorded from a paretic rabbit and a normal rabbit. The retroauricular branch of the facial nerve was stimulated at $20 \mathrm{~Hz}$, and records were taken from the retroauricular muscle using Synax 1100 (NEC). Polyclonal antisera against the $\mathrm{C}$ terminus of the intracellular domain of the MuSK protein were obtained from rabbits injected with a synthetic peptide (CSIHRILQRMCERAEGTVGV). These rabbits remained healthy even after immunization. The specificity of the antiserum was confirmed by Western blot analysis using a lysate of COS-7 cells that were transfected with an expression construct of receptor-type MuSK cDNA. The IgG fraction of the MuSK antiserum was purified by standard methods using protein A-Sepharose (Amersham Biosciences). The concentration of purified IgG was determined with the BCA Protein Assay Kit (Pierce Biotechnology). Normal and paretic rabbits were anesthetized with sodium pentobarbital (Abbott Laboratories; $60 \mathrm{mg} / \mathrm{kg}$, i.p.) and perfused transcardially with 100 $\mathrm{ml}$ of saline, followed by $800 \mathrm{ml}$ of a fixative solution that contained $4 \%$ paraformaldehyde in $0.1 \mathrm{M}$ phosphate buffer ( $\mathrm{pH}$ 7.4). After perfusion, the middle portions of the soleus muscles were removed and immersed in the same fixative at $4^{\circ} \mathrm{C}$ for 24 hours. Cross sections from the soleus muscles of paretic and normal rabbits were stained with $\mathrm{H} \& \mathrm{E}$ for the histological studies and stained with $10 \mathrm{nM}$ rhodamine-conjugated BTX (Invitrogen Corp.) for semi-quantitative measurement of the size and density of AChR clustering at NMJs. AChR clustering was examined at $\times 400$ magnification using a fluorescence microscope (Olympus), and representative images of AChR clusters in random fields were taken with a digital camera (DP70; Olympus). With the unprocessed images, the area and OD of AChR clustering were measured using the Density Slice Command in NIH image analysis software (version 1.62; http://rsb.info.nih.gov/nih-image).

Muscle cell culture. The $\mathrm{C} 2 \mathrm{C} 12$ cell line was obtained from the American Type Culture Collection and used for 3-5 passages. Cells were cultured in 6-well plates as myoblasts in DMEM that contained 10\% FCS. When the cells reached confluence, myotube formation was induced by replacing the medium with DMEM that contained $2 \%$ horse serum. The cells were incubated for 3-4 days, with fresh medium exchange every day, until full differentiation into multinucleated myotubes was observed morphologically. 
AChR clustering assay and immunocytochemical staining. AChR clustering was assayed by growing $\mathrm{C} 2 \mathrm{C} 12$ myotubes in 6-well plates and then treating them for 16 hours with $1 \mathrm{nM}$ agrin (C-Ag4,19), $40 \mathrm{nM}$ laminin-1 (Kouken), $25 \mu \mathrm{g} / \mathrm{ml}$ VVA-B4 (Sigma-Aldrich), or $0.1 \mathrm{U} / \mathrm{ml}$ Clostridium perfringens neuraminidase (Sigma-Aldrich) with or without $10 \mu \mathrm{g} / \mathrm{ml}$ purified MuSK antibodies. The optimal dose of each inducer or MuSK antibodies required for maximal AChR clustering or inhibition, respectively, was determined. Inhibition was blocked by absorption of MuSK antibodies with $6.5 \mathrm{nM}$ MuSK-AP before the cells were treated. The optimal dose of MuSK-AP for neutralization of the MuSK antibodies in the inhibition of AChR clustering assay was determined by serially diluting MuSK-AP protein premixed with antibodies at room temperature, followed by treating the C2C12 myotubes with the mixture. AChR clustering was visualized after incubation with $40 \mathrm{nM}$ rhodamine-conjugated BTX (Invitrogen Corp.) in fusion medium for 1 hour at $37^{\circ} \mathrm{C}$ before fixation. The cells were fixed in $4 \%$ paraformaldehyde for 10 minutes at room temperature. MuSK on the myotubes was then stained using the MuSK antibodies. The myotubes were incubated with the antibodies and rhodamine-conjugated BTX for 1 hour at $37^{\circ} \mathrm{C}$, rinsed, and treated with fluorescein-conjugated goat antimouse antibodies (BioSource International) for 1 hour at $37^{\circ} \mathrm{C}$. The cells were fixed in $4 \%$ paraformaldehyde for 10 minutes at room temperature and then mounted using the SlowFade Antifade Kit (Invitrogen Corp.). The myotubes were examined at $\times 200$ magnification using a fluorescence microscope (Olympus), and photographs of representative images of AChR clusters in random fields were taken using PRESTO NEOPAN400 (Fuji Photo Film Co. Ltd.). The numbers of AChR clusters in the photographs were then counted.

Precipitation assay and immunoblot analysis. Tyrosine phosphorylation of MuSK and AChRs induced by agrin or the antibodies was analyzed by treating $\mathrm{C} 2 \mathrm{C} 12$ myotubes with either $1 \mathrm{nM}$ agrin or $1 \mu \mathrm{g}$ MuSK antibodies (purified by protein A-Sepharose) for 30 minutes with or without $3 \mathrm{nM}$ MuSK-AP. As a control, cells were treated for 30 minutes with antibodies against the $\mathrm{C}$ terminus of the intracellular domain of MuSK (purified by protein A-Sepharose) at a concentration of $1 \mu \mathrm{g} / \mathrm{ml}$. Cultured cells were lysed and solubilized in a protease inhibitor cocktail (Complete EDTA- free; Roche Molecular Biochemicals) with $2 \mathrm{mM}$ sodium orthovanadate. The extracts were immunoprecipitated with antibodies to MuSK, and the resulting precipitates were immunoblotted with a mixture of the antiphosphotyrosine antibodies 4G10 (Upstate USA Inc.) and PY20 (Chemicon International). MuSK was detected by stripping the blots and then reprobing them with the MuSK antibodies. Tyrosine-phosphorylated AChRs were detected by treating the cells with $120 \mathrm{nM}$ biotinylated BTX (Invitrogen Corp.) for 1 hour at $4^{\circ} \mathrm{C}$. The cells were then washed with cold phosphatebuffered saline, followed by lysis and precipitation with streptavidin-coupled agarose beads (Sigma-Aldrich). Phosphotyrosine immunoblotting was performed as described above. AChRs were detected by stripping the blots and then reprobing them for the AChR $\beta$ subunit using a monoclonal antibody (mAb 124; kindly provided by J.M. Lindstrom, University of Pennsylvania, Philadelphia, Pennsylvania, USA) (51).

Statistics. ANOVA was used to analyze the significance of differences between experimental groups. $P$ values less than 0.01 were considered statistically significant.

\section{Acknowledgments}

We thank P. Minick for excellent editorial assistance. This study was supported in part by a grant-in-aid for Scientific Research from the Ministry of Education, Science, and Culture, Japan, and a grant from the Health Science Research Grants for Comprehensive Research on Aging and Health from the Ministry of Health, Labor, and Welfare, Japan. We are also grateful to the staff of the Integrated Center for Science of Ehime University for assistance with the animal care and sequence analysis.

Received for publication March 10, 2004, and accepted in revised form February 7, 2006.

Address correspondence to: Kazuhiro Shigemoto, Department of Preventive Medicine, Ehime University School of Medicine, Sitsukawa, To-on city, Ehime 791-0295, Japan. Phone: 81-89-9605278; Fax: 81-89-960-5279; E-mail: shigemot@m.ehime-u.ac.jp.
1. Lindstrom, J.M., Lennon, V.A., Seybold, M.E., and Whittingham, S. 1976. Experimental autoimmune myasthenia gravis and myasthenia gravis: biochemical and immunochemical aspects. Ann. N. Y. Acad. Sci. 274:254-274.

2. Hoch, W., et al. 2001. Auto-antibodies to the receptor tyrosine kinase MuSK in patients with myasthenia gravis without acetylcholine receptor antibodies. Nat. Med. 7:365-368

3. Evoli, A., et al. 2003. Clinical correlates with antiMuSK antibodies in generalized seronegative myasthenia gravis. Brain. 126:2304-2311.

4. McConville, J., et al. 2004. Detection and characterization of MuSK antibodies in seronegative myasthenia gravis. Ann. Neurol. 55:580-584.

5. Ohta, K., et al. 2004. MuSK antibodies in AChR Ab-seropositive MG vs AChR Ab-seronegative MG. Neurology. 62:2132-2133.

6. Ohta, K., et al. 2005. MuSK Ab described in seropositive MG sera found to be Ab to alkaline phosphatase. Neurology. 65:1988.

7. Sanders, D.B., El-Salem, K., Massey, J.M., McConville, J., and Vincent, A. 2003. Clinical aspects of MuSK antibody positive seronegative MG. Neurology. 60:1978-1980.

8. Yeh, J.H., Chen, W.H., Chiu, H.C., and Vincent, A. 2004. Low frequency of MuSK antibody in generalized seronegative myasthenia gravis among Chinese. Neurology. 62:2131-2132.

9. Zhou, L., et al. 2004. Clinical comparison of muscle-specific tyrosine kinase (MuSK) antibody- positive and -negative myasthenic patients. Muscle Nerve. 30:55-60.

10. Lindstrom, J. 2004. Is "seronegative" MG explained by autoantibodies to MuSK? Neurology. 62:1920-1921.

11. Apel, E.D., Glass, D.J., Moscoso, L.M., Yancopoulos, G.D., and Sanes, J.R. 1997. Rapsyn is required for MuSK signaling and recruits synaptic components to a MuSK-containing scaffold. Neuron. 18:623-635.

12. Marangi, P.A., et al. 2001. Acetylcholine receptors are required for agrin-induced clustering of postsynaptic proteins. EMBO J. 20:7060-7073.

13. Lin, W., et al. 2001. Distinct roles of nerve and muscle in postsynaptic differentiation of the neuromuscular synapse. Nature. 410:1057-1064.

14. Yang, X., et al. 2001. Patterning of muscle acetylcholine receptor gene expression in the absence of motor innervation. Neuron. 30:399-410.

15. DeChiara, T.M., et al. 1996. The receptor tyrosine kinase MuSK is required for neuromuscular junction formation in vivo. Cell. 85:501-512.

16. Gautam, M., et al. 1995. Failure of postsynaptic specialization to develop at neuromuscular junctions of rapsyn-deficient mice. Nature. 377:232-236.

17. Glass, D.J., et al. 1996. Agrin acts via a MuSK receptor complex. Cell. 85:513-523.

18. Cohen, I., Rimer, M., Lomo, T., and McMahan, U.J. 1997. Agrin-induced postsynaptic-like apparatus in skeletal muscle fibers in vivo. Mol. Cell. Neurosci. 9:237-253.

19. Ferns, M., Deiner, M., and Hall, Z. 1996. Agrin- induced acetylcholine receptor clustering in mammalian muscle requires tyrosine phosphorylation. J. Cell Biol. 132:937-944.

20. Ruegg, M.A., et al. 1992. The agrin gene codes for a family of basal lamina proteins that differ in function and distribution. Neuron. 8:691-699.

21. Fu, A.K., et al. 1999. Xenopus muscle-specific kinase: molecular cloning and prominent expression in neural tissues during early embryonic development. Eur. J. Neurosci. 11:373-382.

22. Ganju, P., Walls, E., Brennan, J., and Reith, A.D. 1995. Cloning and developmental expression of Nsk2, a novel receptor tyrosine kinase implicated in skeletal myogenesis. Oncogene. 11:281-290.

23. Ip, F.C., et al. 2000. Cloning and characterization of muscle-specific kinase in chicken. Mol. Cell. Neurosci. 16:661-673.

24. Jennings, C.G., Dyer, S.M., and Burden, S.J. 1993. Muscle-specific trk-related receptor with a kringle domain defines a distinct class of receptor tyrosine kinases. Proc. Natl. Acad. Sci. U. S. A. 90:2895-2899.

25. Valenzuela, D.M., et al. 1995. Receptor tyrosine kinase specific for the skeletal muscle lineage: expression in embryonic muscle, at the neuromuscular junction, and after injury. Neuron. 15:573-584.

26. Zhou, H., Glass, D.J., Yancopoulos, G.D., and Sanes, J.R. 1999. Distinct domains of MuSK mediate its abilities to induce and to associate with postsynaptic specializations. J. Cell Biol. 146:1133-1146.

27. Glass, D.J., et al. 1997. Kinase domain of the musclespecific receptor tyrosine kinase (MuSK) is sufficient 
for phosphorylation but not clustering of acetylcholine receptors: required role for the MuSK ectodomain? Proc. Natl. Acad. Sci. U. S. A. 94:8848-8853.

28. Jones, G., Moore, C., Hashemolhosseini, S., and Brenner, H.R. 1999. Constitutively active MuSK is clustered in the absence of agrin and induces ectopic postsynaptic-like membranes in skeletal muscle fibers. J. Neurosci. 19:3376-3383.

29. Akaaboune, M., Culican, S.M., Turney, S.G., and Lichtman, J.W. 1999. Rapid and reversible effects of activity on acetylcholine receptor density at the neuromuscular junction in vivo. Science. 286:503-507.

30. Akaaboune, M., Grady, R.M., Turney, S., Sanes, J.R., and Lichtman, J.W. 2002. Neurotransmitter receptor dynamics studied in vivo by reversible photo-unbinding of fluorescent ligands. Neuron. 34:865-876.

31. Kong, X.C., Barzaghi, P., and Ruegg, M.A. 2004. Inhibition of synapse assembly in mammalian muscle in vivo by RNA interference. EMBO Rep. 5:183-188.

32. Sugiyama, J.E., Glass, D.J., Yancopoulos, G.D., and Hall, Z.W. 1997. Laminin-induced acetylcholine receptor clustering: an alternative pathway. J. Cell Biol. 139:181-191.

33. Sanes, J.R., and Cheney, J.M. 1982. Lectin binding reveals a synapse-specific carbohydrate in skeletal muscle. Nature. 300:646-647.

34. Martin, P.T., and Sanes, J.R. 1995. Role for a synapse-specific carbohydrate in agrin-induced clustering of acetylcholine receptors. Neuron. 14:743-754.

35. Marangi, P.A., Wieland, S.T., and Fuhrer, C. 2002. Laminin- 1 redistributes postsynaptic proteins and requires rapsyn, tyrosine phosphorylation, and Src and Fyn to stably cluster acetylcholine receptors. J. Cell Biol. 157:883-895.

36. Gautam, M., DeChiara, T.M., Glass, D.J., Yancopoulos, G.D., and Sanes, J.R. 1999. Distinct phenotypes of mutant mice lacking agrin, MuSK, or rapsyn. Brain Res. Dev. Brain Res. 114:171-178.

37. Carpenter, S., and Karpati, G. 1984. Major general pathological reactions and their consequences on skeletal muslce cells. In Pathology of skeletal muscle. S. Carpenter and G. Karpati, editors. Churchill Livingstone Inc. New York, New York, USA. 63-139.

38. Patrick, J., and Lindstrom, J. 1973. Autoimmune response to acetylcholine receptor. Science. 180:871-872.

39. Hopf, C., and Hoch, W. 1998. Dimerization of the muscle-specific kinase induces tyrosine phosphorylation of acetylcholine receptors and their aggregation on the surface of myotubes. J. Biol. Chem. 273:6467-6473.

40. Mittaud, P., Marangi, P.A., Erb-Vogtli, S., and Fuhrer, C. 2001. Agrin-induced activation of acetylcholine receptor-bound Src family kinases requires Rapsyn and correlates with acetylcholine receptor clustering. J. Biol. Chem. 276:14505-14513.

41. Wallace, B.G. 1994. Staurosporine inhibits agrininduced acetylcholine receptor phosphorylation and aggregation. J. Cell Biol. 125:661-668.

42. Burgess, R.W., Nguyen, Q.T., Son, Y.J., Lichtman, J.W., and Sanes, J.R. 1999. Alternatively spliced isoforms of nerve- and muscle-derived agrin: their roles at the neuromuscular junction. Neuron. 23:33-44.
43. Gautam, M., et al. 1996. Defective neuromuscular synaptogenesis in agrin-deficient mutant mice. Cell. 85:525-535.

44. McDearmon, E.L., Combs, A.C., and Ervasti, J.M. 2001. Differential Vicia villosa agglutinin reactivity identifies three distinct dystroglycan complexes in skeletal muscle. J. Biol. Chem. 276:35078-35086.

45. Watty, A., and Burden, S.J. 2002. MuSK glycosylation restrains MuSK activation and acetylcholine receptor clustering. J. Biol. Chem. 277:50457-50462.

46. Gillespie, S.K., Balasubramanian, S., Fung, E.T., and Huganir, R.L. 1996. Rapsyn clusters and activates the synapse-specific receptor tyrosine kinase MuSK. Neuron. 16:953-962.

47. Shigemoto, K., et al. 2000. Identification and characterization of $5^{\prime}$ extension of mammalian agrin cDNA, the exons and the promoter sequences. Biochim. Biophys. Acta. 1494:170-174.

48. Cheng, H.J., Nakamoto, M., Bergemann, A.D., and Flanagan, J.G. 1995. Complementary gradients in expression and binding of ELF-1 and Mek4 in development of the topographic retinotectal projection map. Cell. 82:371-381.

49. Tsim, K.W., Ruegg, M.A., Escher, G., Kroger, S., and McMahan, U.J. 1992. cDNA that encodes active agrin. Neuron. 8:677-689.

50. Rupp, F., et al. 1992. Structure and chromosomal localization of the mammalian agrin gene. J. Neurosci. 12:3535-3544.

51. Tzartos, S.J., Rand, D.E., Einarson, B.L., and Lindstrom, J.M. 1981. Mapping of surface structures of electrophorus acetylcholine receptor using monoclonal antibodies. J. Biol. Chem. 256:8635-8645. 